\title{
ABSTRACTS OF PAPERS
}

\author{
SUBMITTED FOR PRESENTATION TO THE SOCIETY
}

The following papers have been submitted to the Secretary and the Associate Secretaries of the Society for presentation at meetings of the Society. They are numbered serially throughout this volume. Cross references to them in the reports of the meetings will give the number of this volume, the number of this issue, and the serial number of the abstract.

\section{J. H. Blau: A characterization of algebraic fields of prime} characteristic.

A field $F$ is said to be properly generated if it has a proper subring whose quotient field is $F$. It is proved that a field fails to be properly generated if and only if it a finite field or an algebraic extension of a finite field. The general proof makes use of the well-ordering theorem, and hence does not furnish examples of such subrings in particular instances, such as the field of real numbers. (Received January 27,1939.)

\section{S. S. Cairns: Planes transversal to polyhedral manifolds.}

A polyhedral manifold $P^{r}$ is a finite complex of euclidean simplexes in $E^{n}$, each point of which has an $r$-cell for a neighborhood on $P^{r}$. It is a Brouwer manifold if every star of simplexes has a piecewise linear homeomorph in $E^{r}$. Every $P^{r}$ with $r \leqq 3$ is a Brouwer manifold. This is not known to be true for $r>3$. The writer investigates conditions under which one can define, through every point $p$ on $P^{r}$, a continuously varying transversal $(n-r)$-plane, $\pi^{n-r}(p)$. Such a plane is analogous to the normal plane to a differentiable manifold and is characterized by the existence of a neighborhood of $p$ no secant of which is parallel to $\pi^{n-r}(p)$. A necessary condition that $\pi^{n-r}(p)$ exist is that $P^{r}$ be a Brouwer manifold. A sufficient condition, assuming the vertices of $P^{r}$ independent in $E^{n}$, is as follows. Let $\Pi\left(S^{k}\right)$ be the space whose elements are the $(\nu-k)$-planes transversal to a star $S^{k}$ of simplexes on a Brouwer $k$-manifold $(k<r)$ with the vertices independent in $E^{\nu}$. Then every $(r-1-k)$-sphere in any $\Pi\left(S^{k}\right)$, $(k=1, \cdots, r-1)$, must bound a singular $(r-k)$-cell in that $\Pi\left(S^{k}\right)$. This sufficient condition is satisfied for $r \leqq 3$. For $r=4$, it also holds on the basis of a theorem stated by Tietze but apparently not proved in the literature. (Received January 27,1939.)

\section{J. E. Eaton: Associative multiplicative systems.}

This paper is concerned with an algebraic system in which an associate operation is so defined that the product of two non-void subsets is a non-void subset. Conditions on subsets are obtained which insure that they have properties analogous to those of subgroups of groups. Of particular interest are the subsets for which a coset decomposition is possible, and study is made of the homomorphisms so generated. A class of subsets is defined which forms a Dedekind structure. Hence for this class the usual decomposition theorems are valid. All the conditions imposed on subsets in this paper are conditions on the subsets as a whole, rather than on individual members of the subsets. Application is made to the theory of multigroups and also to the theory of ordinary groups. In particular, if the multiplicative system is considered as a multi- 
group, several of the theorems derived represent improvements of known theorems in the theory of multigroups. (Received January 25, 1939.)

112. J. M. Feld: Conformal and inversive invariants of plane curves and horn angles.

E. Kasner has found the unique absolute differential conformal invariant of the third order for horn angles of the first order, that is, curvilinear angles formed by an ordered pair of arcs which pass through a common point in a common direction and have contact of the first order at the common point (Proceedings of the Fifth International Congress of Mathematicians, vol. 2, Cambridge, 1912). Relative conformal invariants of horn angles were determined by Ostrowski (Jahresbericht der deutschen Mathematiker-Vereinigung, vol. 44 (1934)) and independently by Kasner and Comenetz (Proceedings of the National Academy of Sciences, vol. 22 (1936)). In this paper these invariants are shown to be consequences of two formulas, one expressing the ordinary second derivative and the other the Schwarzian derivative in terms of new variables. A new expression for Kasner's absolute horn angle invariant of the third order in terms of Schwarzian derivatives is found. In addition, expressions are found for inversive invariants of curves and curvilinear angles in terms of Schwarzian derivatives and what in this paper are defined to be higher Schwarzian derivatives. (Received January 24, 1939.)

\section{F. C. Gentry: Cremona involutions determined by a pencil of} surfaces.

To each point of a line $d$ are made to correspond $k$ surfaces of a pencil of surfaces of order $n$ containing $d$ as an $(n-2)$-fold line in its base. A generic point $P$ of space determines a surface $S_{n}$ of the pencil. The line joining $P$ to the point of $d$ corresponding to $S_{n}$ meets $S_{n}$ in one further point $Q$, the inverse of $P$ in an involution. The effect on the order of the transformation of the basis curve of the pencil residual to $d$ becoming composite is considered, and the configuration of the $F$ curves of the second kind is determined for each of the cases which arise. (Received January 9, 1939.)

114. Philip Hartman: An asymptotic formula for exponential integrals.

In this paper, conditions for the asymptotic formula (1) $\int_{0}^{1} g(x) \exp \left(-s x^{\alpha}\right) d x$ $\sim C g(+0) s^{-1 / \alpha},|s|=|\sigma+i t| \rightarrow \infty$, are discussed. The conditions for the two regions $|\arg s| \leqq \pi / 2,|\arg s| \leqq \pi / 2-\epsilon$ are of different character. In the half-plane $|\arg s|$ $\leqq \pi / 2$, it is shown that the asymptotic formula (1) holds uniformly if $\alpha>1$ and if $g(x)$ is of bounded variation. In an angular region $|\arg s| \leqq \pi / 2-\epsilon,(1)$ holds uniformly for $\alpha>1$ if it is merely supposed that $g(x)$ is summable and $g(+0)$ exists; if one desires the formula for $\alpha>0$, one must require $|g(x)-g(+0)||\log x| 1 / \alpha \rightarrow 0, x \rightarrow 0$, in addition. The known results (for example, A. Wintner, Proceedings of the $\mathrm{Na}$ tional Academy of Sciences, vol. 20 (1934), pp. 57-62) involving the order of the remainder term may be deduced with an improvement in the conditions from the results obtained here. (Received January 25, 1939.)

\section{Charles Hopkins: An extension of a theorem of Remak.}

Let $G$ be a group with minimal condition for subgroups which are admissible with respect to a set $\Omega$ of (proper) automorphisms of $G$, where $\Omega$ contains all the inner automorphisms of $G$. Then the union $M$ of all minimal admissible subgroups of $G$ is the direct product of a finite number of minimal admissible subgroups; in any two 
such direct decompositions of $M$ the number of components is the same and "corresponding" components are centrally isomorphic. (Received January 23, 1939.)

116. Nathan Jacobson: An application of E. H. Moore's determinant of a hermitian matrix.

It is remarked that E. H. Moore's definition of a determinant holds for hermitian matrices with elements in a general type of ring. As a consequence the following theorem is proved: If $B$ is a matrix of $2 n$ rows and columns with elements in a field of characteristic not equal to 2 such that $R^{-1} B^{\prime} R=B$ where $R$ is any nonsingular skewsymmetric matrix, then the characteristic polynomial of $B$ has the form $[\phi(x)]^{2}$ and $\phi(B)=0$. (Received January 28,1939 .)

\section{Mark Kac: Statistics and power series with gaps.}

Let $\left\{a_{k}\right\}$ be a real bounded sequence for which $\sum a_{k}^{2}=\infty$, and $\left\{n_{k}\right\}$ a sequence of positive integers for which $n_{k} /\left(n_{k}+\cdots+n_{k-1}\right) \rightarrow \infty$ as $k \rightarrow \infty$. Denoting by $F(z)$ the gap series $\sum a_{k} z^{n k},(|z|<1)$, and by $[m(r)]^{2}$ the mean value of $|F(z)|^{2}$ on the circle $|z|=r,(r<1)$, one has the following theorem: If $E$ denotes a rectangle in the complex $F$-plane and $2 \pi r \mu_{r}(E)$ the sum of the lengths of those arcs of the circle $|z|=r$ on which $F\left(r e^{i \phi}\right) / m(r)$ is a point of $E$, then the distribution function $\mu_{r}(E)$ tends, as $r \rightarrow 1$, to a two-dimensional Gaussian distribution function which has density $\pi^{-1} \exp \left\{-\left(u^{2}+v^{2}\right)\right\}$, where $u+i v=F$. (Received January 23, 1939.)

\section{Edward Kasner and J. J. DeCicco: Curvature element trans-} formations which preserve integrable fields.

By a curvature element is meant simply a point of a curve together with the first two derivatives of the curve at the point. A curvature element is given by $\left(x, y, y^{\prime}, y^{\prime \prime}\right)$. A field consists of $\infty^{2}$ curvature elements. If there exist $\infty^{1}$ curves whose curvature elements coincide exactly with the curvature elements of a given field, then the field is termed an integrable field. In this paper, the authors find the group $G$ of all curvature element transformations which convert every integrable field into an integrable field. The fundamental result is that the group $G$ is identical with the group of extended contact transformations of lineal elements. Thus the group $G$ is identical with the group of contact transformations of curvature elements. That is, any transformation of $G$ converts every curve into a curve. The solution of the problem gives a new characteristic property of the group of extended contact transformations of lineal elements (cf. the discussion of an analogous characterization of space contact transformations in a paper appearing in the Duke Mathematical Journal). (Received January 9, 1939.)

119. E. R. Kolchin: On the basis theorem for systems of differential polynomials.

A finite subset $\Phi$ of a system $\Sigma$ of differential polynomials is called a basis of $\Sigma$ if for each differential polynomial $G$ in $\Sigma$ there exists a positive integer $p$ such that $G^{p}$ is in the differential ideal of $\Phi$. The basis is called uniform if a single $p$ can be used for all $G$ 's in $\Sigma$. H. W. Raudenbush has shown (Transactions of this Society, vol. 36 (1934), pp. 361-368) that every system has a basis. In this note an example is given of a perfect differential ideal of differential polynomials which contains no uniform basis. (Received January 6, 1939.) 
120. Saunders MacLane and O. F. G. Schilling: Zero-dimensional branches of rank one on algebraic varieties.

If $L$ is a field of algebraic functions of $n$ variables over a field of constants $K$, a zero-dimensional rank one valuation $V$ of $L$ is a real-valued function $V(a)$ defined on $L$ with the usual valuation properties, and such that $V(a)=0$ for $a$ in $K$, while the residue class field of $V$ is an algebraic extension of $K$. The value group of $V$ is the group of all real numbers $V(a)$ which occur as values. In Zariski's investigations of the resolution of the singularities of algebraic surfaces, one preliminary step is the enumeration of the possible value groups for such a $V$ over a surface $(n=2)$. In this paper the authors investigate the corresponding question for $n$-dimensional varieties. It is proved that the value group either consists of all integral linear combinations of $n$ incommensurable real numbers (a group of rank $n$ ), or else consists of certain linear combinations with rational coefficients of $r$ real numbers, where the rank $r$ is less than $n$. Conversely, any such groups can be realized as value groups, as is shown by two constructions, one using algebraic arguments and one a Puiseux argument. (Received January $23,1939$.

121. Harlan C. Miller: Some methods of characterizing certain atriodic continua.

In this paper the following results are obtained for metric spaces: I. In order that a compact continuum which contains no indecomposable subcontinuum should be an atriodic continuum, every subcontinuum of which is unicoherent, it is necessary and sufficient that every subcontinuum of it be irreducible between two of its points. II. A compact continuum every subcontinuum of which is irreducible between two of its points is atriodic but not necessarily unicoherent. III. If $M$ is a compact continuum, the following statements are equivalent: (1) $M$ is atriodic and every subcontinuum of it is unicoherent. (2) If $A$ and $B$ are two points of $M$, not more than one subcontinuum of $M$ is irreducible between them, and every subcontinuum of $M$ is irreducible between two of its points. (3) Of any three points of $M$, there is one that weakly separates the other two in $M$. (4) If $A, B$, and $C$ are three points of $M$, and $H, K$, and $L$ are three subcontinua of $M$ which are irreducible between $A$ and $B, A$ and $C$, and $B$ and $C$, respectively, one of the continua $H, K, L$ is the sum of the other two. (Received January $13,1939$.

122. D. C. Murdoch and Oystein Ore: Ideal theory in non-associative rings.

This paper contains an axiomatic study of the properties required in a ring in order that the ordinary decomposition theorems for ideals shall hold. It is shown that they depend on the existence of certain weak associative and distributive laws. (Received January 23, 1939.)

\section{Tadasi Nakayama: On Frobeniusean algebras. I.}

Let $A$ be an algebra with a unit element over a field $F . A$ is called Frobeniusean if the right (first) and the left (second) regular representations are equivalent, while $A$ is called quasi-Frobeniusean if the totality of distinct directly indecomposable components of the right regular representation coincides with that of the left regular representation. The present note studies the structure of these types of algebras. The main theorem states: $A$ is Frobeniusean if and only if (i) $l(r(L))=L, r(l(R))=R$, and (ii) $(L: F)+(r(L): F)=(A: F)$ for every left ideal $L$ and right ideal $R$, while $A$ is 
quasi-Frobeniusean if and only if (i) (but not necessarily (ii)) holds for every $L$ and $R$; where $r(S)(l(S)),(S \subset A)$, denotes the set of right (left) annihilators of $S$ in $A$. Frobeniusean algebras have some bearings on principal ideals too. For instance, a necessary and sufficient condition that every two-sided ideal of $A$ can be expressed as $A c=c A$ is that every residue class algebra of $A$ should be Frobeniusean. (Received January 24, 1939.)

\section{Oystein Ore: Chain properties in finite groups.}

This paper contains a study of the properties of non-normal chains of subgroups in finite groups. (Received January 25, 1939.)

\section{H. J. Riblet: Symmetric differential expressions.}

This paper is concerned with the study of the algebraic and differential properties of differential fields obtained by adjoining the roots of an algebraic equation, with coefficients in a differential field $\bar{K}$, to $\bar{K}$. The theorem which serves as a foundation for the paper is as follows. Let $y_{1}, y_{2}, \cdots, y_{n}$ be members of a commutative ring $R_{1}$ in which an operation differentiation obeying the usual sum and product rules is defined. Then any rational integral function of the $y$ 's and their derivatives, with coefficients in a commutative ring $R_{2}$, which is unchanged by all permutations of the $y$ 's is $d$ times a rational integral function $F$ of the elementary symmetric functions of the $y^{\prime}$ s and their derivatives. $F$ has coefficients in $R_{2}$ and $d$ is the reciprocal of a nonnegative power of the discriminant of the $y$ 's. (Received January 25,1939.)

\section{Abraham Sinkov: $A$ note on a paper by J. A. Todd.}

In a recent study of the linear fractional group, J. A. Todd obtained complete definitions for both $\operatorname{LF}\left(2,2^{n}\right)$ and $\operatorname{LF}\left(2, p^{n}\right)$ in terms of $n+2$ generators. A further investigation of these operators shows that, in both cases, two of them, of periods two and three, suffice to generate the entire group. In terms of these two generators, the number of conditions required by Todd's definition is reduced to $n+5$ in the case of $L F\left(2,2^{n}\right)$ and $2 n+6$ in the case of $L F\left(2, p^{n}\right)$. That these restrictions may not all be independent is indicated by the examination of some special cases. Thus it results that $L F\left(2,2^{4}\right)$ and $L F\left(2,3^{3}\right)$ can be completely defined by means of five conditions and $\operatorname{LF}\left(2,2^{5}\right)$ by means of six. All of these latter definitions are new. In addition, the results in connection with $L F\left(2,2^{4}\right)$ are amplified so as to yield necessary and sufficient conditions which must be satisfied by two operators of periods two and three if they are to generate the entire group. (Received January 26, 1939.)

127. S. M. Ulam: On є-isomorphic transformations. Preliminary report.

A transformation $T$ of a group $G_{1}$ into a metric group $G_{2}$ is called an $\epsilon$-isomorphism if $\rho\left(T\left(x_{1} \cdot x_{2}\right), T\left(x_{1}\right) \cdot T\left(x_{2}\right)\right) \leqq \epsilon$ for all $x_{1}, x_{2}$ in $G_{1}, \rho$ denoting the metric distance in $G_{2}$. If $G_{2}$ is compact and if, for every positive $\epsilon$ there is an $\epsilon$-isomorphic transformation of $G_{1}$ into $G_{2}$, there exists a nontrivial homomorphism of $G_{1}$ into $G_{2}$. Continuous $\epsilon$-isomorphisms are studied and their behavior determined for particular groups. (Received January 28, 1939.)

128. Abraham Wald and Jacob Wolfowitz: Confidence limits for continuous distribution functions.

Denote by $X$ a random variable and by $f(x)$ its distribution function, that is, $f(x)$ is the probability that $X<z$. Consider two nonnegative and continuous functions 
$\delta_{1}(y)$ and $\delta_{2}(y)$, defined for $0 \leqq y \leqq 1$, for which $y+\delta_{1}(y)$ and $y-\delta_{2}(y)$ are monotonically increasing in the strict sense. Denote by $\phi_{u}(x)$ the relative frequency of the values less than $x$ in a sample $x_{1}, x_{2}, \cdots, x_{n}$ of the size $n$. It is shown that the probability that $f(x)-\delta_{2}(f) \leqq \phi_{n}(x) \leqq f(x)+\delta_{1}(f)$ shall hold for all values $x$, depends only on $n$ and on the functions $\delta_{1}(y)$ and $\delta_{2}(y)$, provided $f(x)$ is continuous. Denote this probability by $P\left\{n ; \delta_{1}(y) ; \delta_{2}(y)\right\}$. The formula for it has been calculated. If $f(x)$ is unknown but continuous and only a sample of the size $n$ is known, the upper and lower confidence limits of $f(x)$ corresponding to a certain probability level $\alpha,(0<\alpha<1)$, can be determined as follows: The functions $\delta_{1}(y)$ and $\delta_{2}(y)$ are chosen so that $P\left\{n ; \delta_{1}(y)\right.$; $\left.\delta_{2}(y)\right\}=\alpha$. Each of the equations $\delta_{2}\left[\phi_{n}(x)+\Delta_{1}\right]=\Delta_{1}, \delta_{1}\left[\phi_{n}(x)-\Delta_{2}\right]=\Delta_{2}$ has a unique solution in $\Delta_{1}$ and $\Delta_{2}$, respectively. Denote the roots by $\Delta_{1}(x)$ and $\Delta_{2}(x)$. Then the upper confidence limit is given by $\phi_{n}(x)+\Delta_{1}(x)$ and the lower confidence limit by $\phi_{n}(x)-\Delta_{2}(x)$. That is to say if, from a population whose distribution $f(x)$ is unknown but continuous, one draws a sample of size $n$, the probability is $\alpha$ that the sample will have a distribution $\phi_{n}(x)$ for which $\phi_{n}(x)-\Delta_{2}(x) \leqq f(x) \leqq \phi_{n}(x)+\Delta_{1}(x)$. (Received January 25,1939 .)

\section{A. D. Wallace: Non-boundary sets.}

This paper is to appear in volume 45 of this Bulletin. (Received January 21, 1939.)

\section{A. D. Wallace: Some characterizations of interior transforma- tions.}

In this paper the author characterizes interior transformations on connected metric spaces as those continuous transformations for which the inverse of the closure is the closure of the inverse. As a consequence it follows that numerous descriptive point set theoretic properties are invariant under the inverse of an interior transformation. If in addition it is assumed that closed sets map into closed sets, it is shown that the inverse of every point is compact. With the aid of this result it is seen that every normal transformation (as defined by Stoilow) is light, that is, the inverse of every point is totally disconnected. This paper will appear in the American Journal of Mathematics. (Received January 28, 1939.)

\section{J. L. Walsh: On the circles of curvature of the images of circles under a conformal map.}

Under a smooth conformal map of the region $|z|<1$ onto a region $R$ of the extended plane with boundary $B$, no circle of curvature of the image of a circle $|z|$ $=r<2-3^{1 / 2}$ can cut $B$. (Received January 7,1939 .)

\section{Hassler Whitney: On products in a complex. II.}

Duals $D(\sigma)$ to cells $\sigma$ in a complex $K$ are defined. If two systems $D, D^{\prime}$ are in general position, then in each $\sigma^{r+s}$, each pair $D\left(\sigma^{r}\right)$ and $D^{\prime}\left(\sigma^{s}\right)$ has a Kronecker index $\left\{D\left(\sigma^{r}\right) \cdot D^{\prime}\left(\sigma^{s}\right)\right\}_{\sigma^{r+s}}$. These are shown to satisfy the conditions in a previous paper (Annals of Mathematics, (2), vol. 39 (1938), p. 403). Special systems $D, D^{\prime}$ define the products of $\S 6$, loc. cit. "Locally equivalent complexes" are defined, and the products in them considered, which generalizes work of de Rham (Commentarii Mathematici Helvetici, vol. 4, p. 151); in addition, the product of two 1-chains, coefficients modulo 2 , can give a 2-chain with integral coefficients. Some special topics are also considered. This paper is necessary in the general theory of sphere-spaces. (Received January 26, 1939.) 


\section{Norbert Wiener and Brockway McMillan: $A$ new method in} statistical mechanics.

The calculus of the discrete homogeneous chaos is developed and extended to permit applications in the field of statistical mechanics. In particular, as a model for other applications, a discrete homogeneous chaos $M(P ; \alpha)$, representing a distribution of phase points in a six-dimensional phase space, is used as initial conditions for the dynamical equations of an infinite monatomic gas with intermolecular forces. The transformation in time of the chaos in accordance with the laws of dynamics enables one to calculate the time derivatives of linear, quadratic, and higher degree functionals of the chaos. These are at time $t=0$ simply quadratic and higher degree functionals of $M(P ; \alpha)$, and this permits a large class of statistical parameters of the gas to be expanded, at least formally, in power series in the time. The ergodic theorem enables one to calculate these statistical parameters for "almost all" dynamical systems in the ensemble in question by a simple averaging over the $\alpha$ of the initial chaos. Methods of extending these statistical parameters to their equilibrium values at $t=\infty$ will then give information about the physical gas. This extension is made for the mechanical analogue of temperature. (Received January 26,1939.)

\section{Alonzo Church: On the concept of a random sequence.}

The following modification of von Mises' definition of "random sequence" (regellose Folge) is proposed. An infinite sequence $a_{1}, a_{2}, \cdots$, of 0 's and 1 's, for example, is a random sequence (1) if the relative frequency of 1 's among the first $r$ terms of the sequence approaches a limit $p$ as $r \rightarrow \infty$; and (2) if $\phi$ is any effectively calculable function of positive integers in the sense of the author, (American Journal of Mathematics, vol. 58, pp. 345-363), if $b_{1}=1, b_{n+1}=2 b_{n}+a_{n}, c_{n}=\phi\left(b_{n}\right)$, and the integers $n$ such that $c_{n}=1$ form in order of magnitude an infinite sequence $n_{1}, n_{2}, \cdots$, then the relative frequency of 1's among the first $r$ terms of $a_{n_{1}}, a_{n_{2}}, \cdots$ approaches $p$ as $r \rightarrow \infty$. The validity of this rests on the idea that a Spielsystem (von Mises, Wahrscheinlichkeit, Statistik und Wahrheit) should be thought of, not as a function, but as an effective algorithm for the calculation of the values of a function. Since the set of effectively calculable functions is (non-effectively) enumerable, it seems likely that a (non-constructive) existence proof for random sequences in the present sense might result from an extension of the methods of Copeland (American Journal of Mathematics, vol. 58, pp. 181-192). (Received February 2, 1939.)

\section{Douglas Derry: On finite abelian p-groups. I.}

Let $S_{1}, \cdots, S_{n}$ be a set of elements of a finite abelian $p$-group \& $\$$ whose composition series has length $n+1$ such that, for any subgroup $\mathfrak{S}$ of $\$$ with cyclic factor group (J) $/ \mathfrak{S}$, elements $S_{m_{1}}, \cdots, S_{m_{k}}$ exist so that the group series $\left(S_{m_{1}}, \cdots, S_{m_{\vec{\kappa}}}, \mathfrak{W}\right), \cdots$,

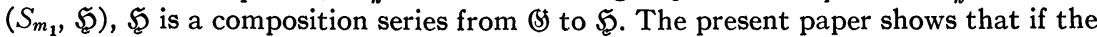
rank of $\$ S$ be 2 , the elements $S_{1}, \cdots, S_{n}$ after a possible rearrangement of order build a composition series $\left(S_{1}, \cdots, S_{n}\right),\left(S_{1}, \cdots, S_{n-1}\right), \cdots,\left(S_{1}\right),(E)$ for $\$$.. (Received January 31,1939 .)

\section{R. J. Duffin and A. C. Schaeffer: $A$ problem in metric diophan-} tine approximation.

By means of continued fractions, Khintchine has shown that if a sequence of positive numbers satisfies the conditions $\sum a_{n}=\infty$ and $n a_{n}$ decreases monotonically, then for almost every real number $x$ there are arbitrarily large integers $n$ and $m$ for which $|n x-m|<a_{n}$. We have found a method of proving this theorem without the use of 
continued fractions. Moreover, we replace the condition $n a_{n}$ monotonically decreasing by the less stringent condition $a_{n}$ monotonically decreasing. Our most general result may be put in this form: let $\left\{b_{i}\right\}$ be any sequence of positive numbers for which $\sum b_{i}=\infty$, and let $\left\{n_{i}\right\}$ be an infinite sequence of integers for which $\phi\left(n_{i}\right) / n_{i}$ has a positive lower bound, where $\phi$ is Euler's $\phi$-function; then for almost every real number $x$ there are arbitrarily large integers $i$ and $m$ for which $\left|n_{i} x-m\right|<b_{i}$. (Received February 13, 1939.)

137. H. T. Engstrom: A note on fundamental systems of symmetric functions.

The author proves that any $n$ polynomials, symmetric in $n$ variables, over a field of characteristic zero form a fundamental system if they are independent and the product of their degrees is less than $2 n !$. The proof is based on Perron's theorem concerning the degree of the dependence relation between $n+1$ polynomials in $n$ variables. (Received February 2, 1939.)

138. L. M. Graves: The Weierstrass condition for multiple integral problems of the calculus of variations.

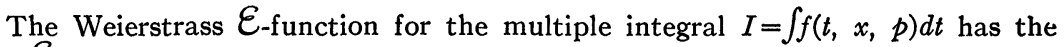
form $\mathcal{E}(t, x, p, P)=f(t, x, P)-f(t, x, p)-\left(P_{\alpha i}-p_{\alpha i}\right) f_{\alpha i}(t, x, p)$, where $t=\left(t_{1}, \cdots, t_{n}\right)$, $x=\left(x_{1}, \cdots, x_{m}\right), p=\left(p_{\alpha i}\right)=\left(\partial x_{i} / \partial t_{\alpha}\right), f_{\alpha i}=\partial f / \partial p_{\alpha i}, i=1, \cdots, m, \alpha=1, \cdots, n$. A necessary condition for a minimum of $I$ is that $\mathcal{E} \geqq 0$ along the minimizing manifold whenever the matrix $\left(P_{\alpha i}-p_{\alpha i}\right)$ has rank one. This has been proved by McShane under very general hypotheses (see Annals of Mathematics, vol. 32 (1931), pp. 578$590)$. The present paper gives an extremely brief and elementary proof, applicable when the minimizing manifold $x_{i}=x_{i}(t)$ has continuous first derivatives $p_{\alpha i}$. For problems in parametric form the integrand $f$ is independent of the variables $t_{\alpha}$, and equals a function $g(x, j)$ of $x_{i}$ and the $n$-rowed minors $j_{\rho}$ of the matrix $\left(p_{\alpha i}\right)$. When these minors $j_{\rho}$ are arranged as an $m$-rowed matrix $k=\left(k_{i \tau}\right)$ in a certain way, it is seen that the rank of $k$ is always $(m-n)$. The matrix $\left(P_{\alpha i}-p_{\alpha i}\right)$ has rank one if and only if the matrix $\left(k_{i r}, K_{i \sigma}\right)$ has rank $(m-n+1)$. It is necessary for a minimum of $I$ that $\mathcal{E}(x, j, J)=g(x, J)-g(x, j)-\left(J_{\rho}-j_{\rho}\right) g_{\rho} \geqq 0$ for all $J_{\rho}$ such that this condition is satisfied. This result was proved directly for parametric problems for the cases $n=1$ and $n=m-1$ by McShane (ibid., pp. 723-733). (Received January 30, 1939.)

139. Nathan Jacobson: The fundamental theorem of the Galois theory for quasi-fields.

Let $\mathrm{P}$ be an arbitrary quasi-field and $\$$ a finite group of $n$ outer automorphisms in P. If $\Phi$ is the set of invariant elements, either order $(P: \Phi)=n$ and there is a (1-1) correspondence between the subgroups of $\$$ and the sub-quasi-fields $\Sigma$ between $\Phi$ and $\mathbf{P}$. The proof of this extension of the fundamental theorem of Galois theory is obtained by considering the ring of operators $\sum S \xi_{S}$ acting in $\mathrm{P}$ where $S \varepsilon \&$ and $\xi$ is the operator $\alpha \rightarrow \alpha \xi$. The theorem has applications to division algebras. The method used gives also the following extension of a well known theorem of Hilbert: Let $U_{S}$ be a set of matrices corresponding to the $S$ 's of \&s such that $U_{1}=1$ and $U_{T} U_{S} T=U_{S T}$, then there exists a matrix $A$ such that $U_{S}=A^{-1} A^{S}$. (Received February 14,1939.)

140. D. H. Lehmer: On the remainders and convergence of the series for the partition function.

The two series under discussion are the asymptotic series of Hardy and Ramanujan (1917) and the convergent series of Rademacher (1937) for the number $p(n)$ of 
unrestricted partitions of $n$. (See, for instance, Transactions of this Society, vol. 43 (1938), pp. 271-295.) The writer has given estimates for the remainders $R_{1}(n, N)$, $R_{2}(n, N)$ after $N$ terms of these respective series of such a form that in either case $R_{i}\left(n, \alpha n^{1 / 2}\right)=O\left(n^{-1 / 3}\right)$, as compared with the previous result of $O\left(n^{-1 / 4}\right)$. In the present paper the author obtains more precise estimates for which $R_{i}\left(n, \alpha n^{1 / 2}\right)=O\left(n^{-1 / 2} \log n\right)$, a result which is, in a sense, the best possible. The divergence of the Hardy-Ramanujan series was proved by the writer in 1937 by a method which did not remove the possibility of the series oscillating between fixed limits. In this paper the author gives a new proof of divergence showing that the terms of the series are in fact unbounded. For the Rademacher series it is proved that the $k$ th term exceeds $1 / k^{2}$ in absolute value for infinitely many $k$ 's. A brief discussion is given of the function $p(-n)$ defined by the Rademacher series and conjectured to be identically zero. It is shown that $p(-n)=O\left(n^{-1 / 2} \log n\right)$ and that the remainder of its series after $N$ terms is $O\left(N^{-1} \log N\right)$, for every $n$. (Received February 4,1939.)

\section{Norman Levinson: An inequality in function theory.}

Let $M(x)$ be an even function of $x$, monotone decreasing for positive $x$. Let $F(z)$, $z=x+i y$, be analytic in the square $(|y|<1,|x|<1)$, and let $|F(x+i y)|$ be less than $M(x)$ in this square. A necessary and sufficient condition that there exist a constant $C$ depending only on $M(x)$ and $\delta>0$ such that $|F(x+i y)|<C,(|x|<1-\delta,|y|<1-\delta)$, is that $\int_{0}^{1} \log \log M(x) d x<\infty$. (Received February 15, 1939.)

\section{R. E. O'Connor: Quadratic and linear congruence.}

The number $N(m)$ of simultaneous solutions of the integral congruences, $\sum a_{i j} x_{i} x_{j} \equiv r, \sum b_{i} x_{i} \equiv s(\bmod m),(i, j=1,2, \cdots, n)$, is given for $m$ odd, prime to the g.c.d. of each set of coefficients, and either square-free or at least containing no prime factors of a certain class to a degree higher than the first. An application is made to 3-dimensional vectors defined with respect to a quadratic form. (Received February 13, 1939.)

\section{L. F. Ollmann: On joining finite subsets of a planar Peano} space by simple closed curves.

Let $K$ be a finite subset of a Peano space $M$ in the euclidean plane such that no two points of $K$ may be separated by the omission of any two points of $M$. Then: (1) If $K$ consists of no more than 5 points, there is always a simple closed curve $J$ such that $M \supset J \supset K$. (2) Two examples are given to show that (1) is not true for 6 points. (In one example, each point of $M$ is of Menger order $c$, while in the other each point is of order less than or equal to 4.) (3) For any $n$ a set $M$ is constructed containing $2 n$ points which cannot be joined by $n-2$ mutually exclusive simple closed curves in $M$. (4) For any $n$ a cubic graph $M$ is constructed in which the vertices cannot be joined by $n$ simple closed curves (not necessarily mutually exclusive). (5) An example is given of a set $M$, which is a triangular graph (all faces are triangles), such that the vertices cannot be joined by a simple closed curve. (Received February 16, 1939.)

\section{J. F. Ritt: On ideals of differential polynomials.}

Let $\Sigma$ be an ideal of differential polynomials. Let the manifold of $\Sigma$ be separable into submanifolds none of which has a solution in common with any other. It is proved that $\Sigma$ can be represented in one and in only one way as the intersection of ideals whose manifolds are the various submanifolds. (Received February 2,1939.) 
145. A. C. Schaeffer: The Fourier-Stieltjes coefficients of a function of bounded variation.

Let $F(x)$, defined in the interval $(-\pi, \pi)$, be a continuous function which is of bounded variation but is not absolutely continuous, and let $c_{n}(F)$ denote its FourierStieltjes coefficients. In a recent paper (American Journal of Mathematics, vol. 60 (1938), pp. 513-522) Wiener and Wintner, improving an earlier result of Littlewood, have shown that, if $\gamma$ is any positive number less than $1 / 2$, there exists an $F(x)$ such that $c_{n}(F)=O\left(n^{-\gamma}\right)$. In this paper the method of Wiener and Wintner is used to show that if $r(n)$ is any function of $n$ which tends to infinity as $n$ tends to infinity, then there exists an $F(x)$ such that $c_{n}(F)=O\left(r(n) n^{-1 / 2}\right)$. This function satisfies the additional condition, as have earlier examples, that its derivative vanishes almost everywhere. (Received February 11, 1939.)

146. W. E. Sewell: Integral approximation and continuity in the real domain.

In this paper the author studies the relation between the degree of approximation to a periodic function $f(\theta)$ by trigonometric sums in the sense of least $p$ th powers and the continuity properties of $f(\theta)$. (Received February 10,1939.)

147. W. E. Sewell: Integral approximation and cortinuity in the complex domain.

In this paper the author studies the relation between degree of approximation by polynomials in the sense of least $p$ th powers measured by a line integral taken over a rectifiable Jordan curve $C$ and the continuity properties of the function approximated on the curve $C_{R}$ in the complex domain. (Received February 10,1939.)

148. H. C. Trimble: On the ring of matrices commutative with a given matrix.

Let $R(A)$ be the ring of matrices commutative with a given $n$th order square matrix $A$ with elements in a commutative field $K$. Two isomorphisms of a certain class of isomorphisms (introduced by.M. H. Ingraham and the author and similar to an isomorphism given by $\mathrm{P}$. L. Trump) are established. In either case, $R(A)$ is shown to be isomorphic to a system of classes of matrices of lower order whose elements are polynomials in an indeterminate $\lambda$, and whose rows are reduced modulo certain factors of the minimal polynomial of $A$. By using these isomorphisms, $R(A)$ is determined rationally, well known properties of $R(A)$ are proved simply, and new results are obtained. The bulk of the paper is an outline of a rational attack upon the similarity problem in $R(A)$. The methods are believed suggestive, and though the theory is incomplete in one place, no example has been found to escape the methods used. The case in which $A$ has at most two invariant factors is solved completely. (Received January $26,1939$.

149. E. P. Vance: Generalizations of non-alternating and non-separating transformations.

G. T. Whyburn (American Journal of Mathematics, vol. 56 (1934), pp. 294-302) and J. F. Wardwell (Duke Journal of Mathematics, vol. 2 (1936), pp. 745-750) have considered non-alternating and non-separating transformations in recent papers. It is possible to obtain interesting results by weakening the conditions of these transforma- 
tions and also of completely non-alternating and completely non-separating transformations. A continuous transformation will be defined to be weakly non-alternating provided $T^{-1}(x)$ does not separate $T^{-1}(y)$ between any two points $u$ and $v$ of $T^{-1}(y)$ unless a single point does. Similar definitions can be made for the three other transformations. In this paper these four transformations are characterized, factor and product theorems are proved, and the transformations are applied to locally connected continua and to special curves and surfaces. (Received February 16,1939.)

150. Abraham Wald: Lower and upper limits of a distribution function determined by moments and inequalities satisfied by moments.

Denote by $X$ a random variable and by $P(\alpha<X<\beta)$ the probability that $\alpha<X<\beta$. For any positive integer $r$ the expected value $E\left|X-x_{0}\right|^{r}$ of $\left|X-x_{0}\right|^{r}$ is called the absolute moment of the order $r$ about $x_{0}$. Denote by $i_{1}, i_{2}, \cdots, i_{i} j$ different positive integers and by $M_{i_{1}}, \cdots, M_{i_{j}}$ the absolute moments of the order $i_{1}, \cdots, i_{j}$ of a certain random variable $X$ about $x_{0}$. For any $d>0$ the greatest lower bound $a_{d}$ and the least upper bound $b_{d}$ of the probabilities $P\left(-d<Y-x_{0}<d\right)$, formed for all random variables $Y$ for which $E\left|Y-x_{0}\right| i_{\nu}=E\left|X-x_{0}\right| i_{\nu}=M_{i \nu}, \quad(\nu=1, \cdots, j)$, are calculated. This is a generalization of the Tshebysheff-Markoff inequalities which give the upper and lower limits in question only if $i_{1}=1, \cdots, i_{j}=j$ and only for certain special values of $d$. In this paper is given also the complete system of inequalities satisfied by absolute moments. That is to say, for any $j$ different positive integers $i_{1}, \cdots, i_{j}$ the necessary and sufficient conditions are given which must be satisfied by $j$ numbers $c_{1}, \cdots, c_{j}$ in order that a random variable $X$ should exist of which the $i_{\nu}$ th absolute moment about $x_{0}$ is equal to $c_{\nu},(\nu=1, \cdots, j)$. (Received February 2, 1939.)

\section{Max Zorn: Continuous groups and Schwarz' lemma.}

This paper contains a discussions of Schwarz' lemma with elementary topological methods. The results are of use for the topological characterization of the set of all conformal mappings within a Riemann surface. (Received February 7, 1939.) 